\title{
Halpern iteration for strongly quasinonexpansive mappings on a geodesic space with curvature bounded above by one
}

\author{
Yasunori Kimura ${ }^{1 *}$ and Kenzi Satô
}

"Correspondence:

yasunori@is.sci.toho-u.ac.jp

1 Department of Information

Science, Toho University, Miyama, Funabashi, Chiba 274-8510, Japan

Full list of author information is

available at the end of the article

\begin{abstract}
In this paper, we deal with the Halpern iterative scheme for a strongly quasinonexpansive mapping in the setting of a complete geodesic space with curvature bounded above by one. Our result can be applied to the image recovery problem. We also consider the approximation of a fixed point of a nonexpansive mapping and obtain convergence theorems, one of which is a supplement of the result by Piątek with an additional sufficient condition.
\end{abstract}

MSC: $47 \mathrm{H} 09$

Keywords: CAT(1) space; Halpern iteration; strongly quasinonexpansive; $\Delta$-demiclosed; fixed point

\section{Introduction}

Halpern's iterative method [1] is one of the most effective methods to find a fixed point of a nonexpansive mapping, which guarantees strong convergence of the approximating sequence. A remarkable result for nonlinear mappings was obtained by Wittmann [2] in the setting of Hilbert spaces. Since then, it has been investigated by a large number of researchers, and they have obtained different types of strong convergence theorems for nonexpansive mappings and their variations.

On the other hand, the notion of a strongly nonexpansive mapping was first proposed by Bruck and Reich [3] as a generalization of firmly nonexpansive mappings. This mapping was later generalized to a strongly quasinonexpansive mapping by Bruck [4]. We propose a new definition of this mapping in the framework of CAT(1) space by imposing a natural bound on the diameter of the space.

The first result of convergence of the Halpern iteration on a complete CAT( 0$)$ space was obtained by Saejung [5] for nonexpansive mappings, and a similar result in the setting of a complete CAT(1) space was proposed by Piatek [6]. The combination of the Halpern iteration and strongly quasinonexpansive mappings was made recently. See $[7,8]$ and others.

In this paper, we deal with the Halpern iterative scheme for a strongly quasinonexpansive mapping in the setting of a complete CAT(1) space. Then we show that the main result can be applied to the problem of image recovery. We also consider the approximation of a fixed point of a nonexpansive mapping. We point out that the proof of the result by Piątek is not

(c) 2013 Kimura and Satô; licensee Springer. This is an Open Access article distributed under the terms of the Creative Commons Attribution License (http://creativecommons.org/licenses/by/2.0), which permits unrestricted use, distribution, and reproduction in any medium, provided the original work is properly cited. 
sufficient and we supplement it with an additional sufficient condition for the convergence of the iterative sequence.

\section{Preliminaries}

Let $X$ be a metric space. An element $z$ of $X$ is said to be an asymptotic center of a sequence $\left\{x_{n}\right\}$ in $X$ if

$$
\limsup _{n \rightarrow \infty} d\left(x_{n}, z\right)=\inf _{x \in X} \limsup _{n \rightarrow \infty} d\left(x_{n}, x\right) .
$$

Moreover, $\left\{x_{n}\right\}$ is said to be $\Delta$-convergent and $z$ is said to be its $\Delta$-limit if $z$ is the unique asymptotic center of any subsequences of $\left\{x_{n}\right\}$.

A geodesic with endpoints $x, y \in X$ is defined as an isometric mapping from the closed segment $[0, l]$ of real numbers to $X$ whose image connects $x$ and $y$. If a geodesic exists for every $x, y \in X$, then $X$ is called a geodesic space.

For a triangle $\Delta(x, y, z)$ in a geodesic space $X$ satisfying $d(y, z)+d(z, x)+d(x, y)<2 \pi$, we can find the comparison triangle $\Delta(\bar{x}, \bar{y}, \bar{z})$ in $\mathbb{S}^{2}$, that is, each corresponding edge has the same length as that of the original triangle. If every two points $p, q$ on the edges of any $\triangle(x, y, z)$ and their corresponding points $\bar{p}, \bar{q}$ satisfy that

$$
d(p, q) \leq d_{\mathbb{S}^{2}}(\bar{p}, \bar{q})
$$

we call $X$ a CAT(1) space, where $d_{\mathbb{S}^{2}}$ is the spherical metric on $\mathbb{S}^{2}$.

In this paper, we deal with only CAT(1) spaces; however, we remark that all the results can be easily generalized to $\mathrm{CAT}(\kappa)$ spaces with positive $\kappa$ by changing the scale of the space.

For two points $x, y$ in a CAT(1) space $X$ with $d(x, y)<\pi$ and $t \in[0,1]$, we denote by $t x \oplus(1-t) y$ the point $z$ on a geodesic segment between $x$ and $y$ such that $d(y, z)=t d(x, y)$ and $d(x, z)=(1-t) d(x, y)$. A subset $C$ of $X$ is said to be $\pi$-convex if $t x \oplus(1-t) y$ belongs to $C$ for every $x, y \in C$ with $d(x, y)<\pi$ and $t \in[0,1]$.

We refer to [9] for more details on geodesic spaces including CAT(1) spaces.

For three points $x, y, z$ in a CAT(1) space $X$ with $d(y, z)+d(z, x)+d(x, y)<2 \pi$ and $t \in[0,1]$, we know that the following inequality holds [10]:

$$
\cos d(x, v) \sin d(y, z) \geq \cos d(x, y) \sin (t d(y, z))+\cos d(x, z) \sin ((1-t) d(y, z)),
$$

where $v=t y \oplus(1-t) z$. This simple inequality plays a very important role in this paper.

Let $X$ be a complete CAT(1) space, $C$ a nonempty closed $\pi$-convex subset of $X$ and suppose that $d(x, C)=\inf _{y \in C} d(x, y)<\pi / 2$ for every $x \in X$. Then we can define the metric projection $P_{C}$ from $X$ onto $C$; that is, for every $x \in X, P_{C} x \in C$ is the unique point satisfying

$$
d\left(x, P_{C} x\right)=\inf _{y \in C} d(x, y)
$$

Let $X$ be a CAT(1) space. Let $T: X \rightarrow X$ and suppose that the set $F(T)=\{x \in X: x=T x\}$ of fixed points is not empty. Then $T$ is said to be quasinonexpansive if $d(T x, p) \leq d(x, p)$ for every $x \in X$ and $p \in F(T)$. T is said to be strongly quasinonexpansive if it is quasinonexpansive, and for every $p \in F(T)$ and every sequence $\left\{x_{n}\right\}$ in $X$ satisfying that $\sup _{n \in \mathbb{N}} d\left(x_{n}, p\right)<$ 
$\pi / 2$ and $\lim _{n \rightarrow \infty}\left(\cos d\left(x_{n}, p\right) / \cos d\left(T x_{n}, p\right)\right)=1$, it follows that $\lim _{n \rightarrow \infty} d\left(x_{n}, T x_{n}\right)=0 . T$ is said to be $\Delta$-demiclosed if for any $\Delta$-convergent sequence $\left\{x_{n}\right\}$ in $X$, its $\Delta$-limit belongs to $F(T)$ whenever $\lim _{n \rightarrow \infty} d\left(x_{n}, T x_{n}\right)=0$.

The following lemmas are important for our main result.

Lemma 2.1 (Xu [11]) Let $\left\{s_{n}\right\},\left\{t_{n}\right\}$ and $\left\{u_{n}\right\}$ be sequences of real numbers such that $s_{n} \geq 0$ and $u_{n} \geq 0$ for every $n \in \mathbb{N}$, $\limsup _{n \rightarrow \infty} t_{n} \leq 0$, and $\sum_{n=0}^{\infty} u_{n}<\infty$. Let $\left\{\gamma_{n}\right\}$ be a sequence in $[0,1]$ such that $\sum_{n=0}^{\infty} \gamma_{n}=\infty$. If $s_{n+1} \leq\left(1-\gamma_{n}\right) s_{n}+\gamma_{n} t_{n}+u_{n}$ for every $n \in \mathbb{N}$, then $\lim _{n \rightarrow \infty} s_{n}=0$.

Lemma 2.2 (Saejung-Yotkaew [12]) Let $\left\{s_{n}\right\}$ and $\left\{t_{n}\right\}$ be sequences of real numbers such that $s_{n} \geq 0$ for every $n \in \mathbb{N}$. Let $\left\{\beta_{n}\right\}$ be a sequence in $] 0,1\left[\right.$ such that $\sum_{n=0}^{\infty} \beta_{n}=\infty$. Suppose that $s_{n+1} \leq\left(1-\beta_{n}\right) s_{n}+\beta_{n} t_{n}$ for every $n \in \mathbb{N}$. If $\limsup _{k \rightarrow \infty} t_{n_{k}} \leq 0$ for every subsequence $\left\{n_{k}\right\}$ of $\mathbb{N}$ satisfying $\liminf _{k \rightarrow \infty}\left(s_{n_{k}+1}-s_{n_{k}}\right) \geq 0$, then $\lim _{n \rightarrow \infty} s_{n}=0$.

Lemma 2.3 (He-Fang-Lopez-Li [13]) Let $X$ be a complete CAT(1) space and $p \in X$. If a sequence $\left\{x_{n}\right\}$ in $X$ satisfies that $\limsup _{n \rightarrow \infty} d\left(x_{n}, p\right)<\pi / 2$ and that $\left\{x_{n}\right\}$ is $\Delta$-convergent to $x \in X$, then $d(x, p) \leq \liminf _{n \rightarrow \infty} d\left(x_{n}, p\right)$.

\section{Main result}

As the main theorem of this paper, we prove strong convergence of the iterative sequence to a fixed point of a strongly quasinonexpansive mapping. We adopt the Halpern iterative scheme to generate the sequence. We begin with the following basic lemma, which is one of the main tools for our results.

Lemma 3.1 Let $X$ be a CAT(1) space such that $d\left(v, v^{\prime}\right)<\pi$ for every $v, v^{\prime} \in X$. Let $\alpha \in[0,1]$ and $u, y, z \in X$. Then

$$
\begin{aligned}
1- & \cos d(\alpha u \oplus(1-\alpha) y, z) \\
& \leq(1-\beta)(1-\cos d(y, z))+\beta\left(1-\frac{\cos d(u, z)}{\sin d(u, y) \tan \left(\frac{\alpha}{2} d(u, y)\right)+\cos d(u, y)}\right),
\end{aligned}
$$

where

$$
\beta= \begin{cases}1-\frac{\sin ((1-\alpha) d(u, y))}{\sin d(u, y)} & (u \neq y) \\ \alpha & (u=y) .\end{cases}
$$

Proof It is obvious if $u=y$. Otherwise, from the inequality

$$
\cos d(\alpha u \oplus(1-\alpha) y, z) \geq \frac{\sin (\alpha d(u, y))}{\sin d(u, y)} \cos d(u, z)+(1-\beta) \cos d(y, z)
$$

we have that

$$
\begin{aligned}
1 & -\cos d(\alpha u \oplus(1-\alpha) y, z) \\
& \leq(1-\beta)(1-\cos d(y, z))+\beta\left(1-\frac{\sin (\alpha d(u, y))}{\beta \sin d(u, y)} \cos d(u, z)\right) .
\end{aligned}
$$


We also have that

$$
\begin{aligned}
\frac{\sin (\alpha d(u, y))}{\beta \sin d(u, y)} & =\frac{\sin (\alpha d(u, y))}{\sin d(u, y)-\sin ((1-\alpha) d(u, y))} \\
& =\frac{\sin (\alpha d(u, y))}{\sin d(u, y)(1-\cos (\alpha d(u, y)))+\cos d(u, y) \sin (\alpha d(u, y))} \\
& =\frac{1}{\sin d(u, y) \tan \left(\frac{\alpha}{2} d(u, y)\right)+\cos d(u, y)}
\end{aligned}
$$

and hence we obtain the desired result.

Remark On the same assumption, we have

$$
\cos d(\alpha u \oplus(1-\alpha) y, z) \geq \alpha \cos d(u, z)+(1-\alpha) \cos d(y, z)
$$

Indeed, it holds from the first inequality of the proof above together with

$$
\frac{\sin (\alpha d(u, y))}{\sin d(u, y)} \geq \alpha \quad \text { and } \quad \frac{\sin ((1-\alpha) d(u, y))}{\sin d(u, y)} \geq 1-\alpha .
$$

Now, we show the main theorem.

Theorem 3.2 Let $X$ be a complete CAT(1) space such that $d\left(v, v^{\prime}\right)<\pi / 2$ for every $v, v^{\prime} \in X$. Let $T: X \rightarrow X$ be a strongly quasinonexpansive and $\Delta$-demiclosed mapping, and suppose that $F(T) \neq \emptyset$. Let $\left\{\alpha_{n}\right\}$ be a real sequence in $] 0,1\left[\right.$ such that $\lim _{n \rightarrow \infty} \alpha_{n}=0$ and $\sum_{n=0}^{\infty} \alpha_{n}=$ $\infty$. For given points $u, x_{0} \in X$, let $\left\{x_{n}\right\}$ be the sequence in $X$ generated by

$$
x_{n+1}=\alpha_{n} u \oplus\left(1-\alpha_{n}\right) T x_{n}
$$

for $n \in \mathbb{N}$. Suppose that one of the following conditions holds:

(a) $\sup _{v, v^{\prime} \in X} d\left(v, v^{\prime}\right)<\pi / 2$;

(b) $d\left(u, P_{F(T)} u\right)<\pi / 4$ and $d\left(u, P_{F(T)} u\right)+d\left(x_{0}, P_{F(T)} u\right)<\pi / 2$;

(c) $\sum_{n=0}^{\infty} \alpha_{n}^{2}=\infty$.

Then $\left\{x_{n}\right\}$ converges to $P_{F(T)} u$.

Proof Let $p=P_{F(T)} u$ and let

$$
\begin{aligned}
& s_{n}=1-\cos d\left(x_{n}, p\right), \\
& t_{n}=1-\frac{\cos d(u, p)}{\sin d\left(u, T x_{n}\right) \tan \left(\frac{\alpha_{n}}{2} d\left(u, T x_{n}\right)\right)+\cos d\left(u, T x_{n}\right)}, \\
& \beta_{n}= \begin{cases}1-\frac{\sin \left(\left(1-\alpha_{n}\right) d\left(u, T x_{n}\right)\right)}{\sin d\left(u, T x_{n}\right)} & \left(u \neq T x_{n}\right), \\
\alpha_{n} & \left(u=T x_{n}\right)\end{cases}
\end{aligned}
$$

for $n \in \mathbb{N}$. Then, since $T$ is quasinonexpansive, it follows from Lemma 3.1 that

$$
s_{n+1} \leq\left(1-\beta_{n}\right)\left(1-\cos d\left(T x_{n}, p\right)\right)+\beta_{n} t_{n} \leq\left(1-\beta_{n}\right) s_{n}+\beta_{n} t_{n}
$$


for every $n \in \mathbb{N}$. We also have that

$$
\begin{aligned}
\cos d\left(x_{n+1}, p\right) & =\cos d\left(\alpha_{n} u \oplus\left(1-\alpha_{n}\right) T x_{n}, p\right) \\
& \geq \alpha_{n} \cos d(u, p)+\left(1-\alpha_{n}\right) \cos d\left(T x_{n}, p\right) \\
& \geq \alpha_{n} \cos d(u, p)+\left(1-\alpha_{n}\right) \cos d\left(x_{n}, p\right) \\
& \geq \min \left\{\cos d(u, p), \cos d\left(x_{n}, p\right)\right\}
\end{aligned}
$$

for all $n \in \mathbb{N}$. Thus we obtain that

$$
\cos d\left(x_{n}, p\right) \geq \min \left\{\cos d(u, p), \cos d\left(x_{0}, p\right)\right\}=\cos \max \left\{d(u, p), d\left(x_{0}, p\right)\right\}>0
$$

for all $n \in \mathbb{N}$ and hence $\sup _{n \in \mathbb{N}} d\left(x_{n}, p\right) \leq \max \left\{d(u, p), d\left(x_{0}, p\right)\right\}<\pi / 2$.

Now, we see that each of the conditions (a), (b) and (c) implies that $\sum_{n=0}^{\infty} \beta_{n}=\infty$. For the cases of (a) and (b), let $M=\sup _{n \in \mathbb{N}} d\left(u, T x_{n}\right)$. Then we show that $M<\pi / 2$. For (a), it is trivial. For (b), since $\sup _{n \in \mathbb{N}} d\left(x_{n}, p\right) \leq \max \left\{d(u, p), d\left(x_{0}, p\right)\right\}$, we have that

$$
\begin{aligned}
M & \leq \sup _{n \in \mathbb{N}}\left(d(u, p)+d\left(T x_{n}, p\right)\right) \\
& \leq \sup _{n \in \mathbb{N}}\left(d(u, p)+d\left(x_{n}, p\right)\right) \\
& \leq \max \left\{2 d(u, p), d(u, p)+d\left(x_{0}, p\right)\right\} \\
& <\frac{\pi}{2} .
\end{aligned}
$$

So, in each case of (a) and (b), we have

$$
\begin{aligned}
\beta_{n} & \geq 1-\frac{\sin \left(\left(1-\alpha_{n}\right) M\right)}{\sin M} \\
& =\frac{2}{\sin M} \sin \left(\frac{\alpha_{n}}{2} M\right) \cos \left(\left(1-\frac{\alpha_{n}}{2}\right) M\right) \\
& \geq \alpha_{n} \cos M .
\end{aligned}
$$

Since $\sum_{n=0}^{\infty} \alpha_{n}=\infty$, it follows that $\sum_{n=0}^{\infty} \beta_{n}=\infty$. For the case of (c), we have that

$$
\beta_{n} \geq 1-\sin \frac{\left(1-\alpha_{n}\right) \pi}{2}=1-\cos \frac{\alpha_{n} \pi}{2} \geq \frac{\alpha_{n}^{2} \pi^{2}}{8}
$$

for every $n \in \mathbb{N}$. Therefore, from the condition (c) we have that $\sum_{n=0}^{\infty} \beta_{n}=\infty$.

For any subsequence $\left\{s_{n_{j}}\right\}$ of $\left\{s_{n}\right\}$ satisfying that $\liminf _{j \rightarrow \infty}\left(s_{n_{j}+1}-s_{n_{j}}\right) \geq 0$, we have that

$$
\begin{aligned}
0 & \leq \liminf _{j \rightarrow \infty}\left(s_{n_{j}+1}-s_{n_{j}}\right) \\
& =\liminf _{j \rightarrow \infty}\left(\cos d\left(x_{n_{j}}, p\right)-\cos d\left(x_{n_{j}+1}, p\right)\right) \\
& \leq \liminf _{j \rightarrow \infty}\left(\cos d\left(x_{n_{j}}, p\right)-\left(\alpha_{n_{j}} \cos d(u, p)+\left(1-\alpha_{n_{j}}\right) \cos d\left(T x_{n_{j}}, p\right)\right)\right) \\
& =\liminf _{j \rightarrow \infty}\left(\cos d\left(x_{n_{j}}, p\right)-\cos d\left(T x_{n_{j}}, p\right)\right)
\end{aligned}
$$




$$
\begin{aligned}
& \leq \limsup _{j \rightarrow \infty}\left(\cos d\left(x_{n_{j}}, p\right)-\cos d\left(T x_{n_{j}}, p\right)\right) \\
& \leq 0
\end{aligned}
$$

Thus we have that $\lim _{j \rightarrow \infty}\left(\cos d\left(x_{n_{j}}, p\right)-\cos d\left(T x_{n_{j}}, p\right)\right)=0$. Using the inequality $\sup _{n \in \mathbb{N}} d\left(T x_{n}, p\right)<\pi / 2$, we also have $\lim _{j \rightarrow \infty}\left(\cos d\left(x_{n_{j}}, p\right) / \cos d\left(T x_{n_{j}}, p\right)\right)=1$. Since $T$ is strongly quasinonexpansive, it follows that $\lim _{j \rightarrow \infty} d\left(x_{n_{j}}, T x_{n_{j}}\right)=0$. Let $\left\{v_{k}\right\}$ be a $\Delta$ convergent subsequence of $\left\{x_{n_{j}}\right\}$ such that $\lim _{k \rightarrow \infty} d\left(u, v_{k}\right)=\liminf _{j \rightarrow \infty} d\left(u, x_{n_{j}}\right)$. Then, since $T$ is $\Delta$-demiclosed and $\lim _{k \rightarrow \infty} d\left(v_{k}, T v_{k}\right)=0$, the $\Delta$-limit $z$ of $\left\{v_{k}\right\}$ belongs to $F(T)$. Using Lemma 2.3 and the definitions of the $\Delta$-limit and the metric projection, we have that

$$
\liminf _{j \rightarrow \infty} d\left(u, T x_{n_{j}}\right)=\liminf _{j \rightarrow \infty} d\left(u, x_{n_{j}}\right)=\lim _{k} d\left(u, v_{k}\right) \geq d(u, z) \geq d(u, p) .
$$

Therefore, we obtain that

$$
\begin{aligned}
\limsup _{j \rightarrow \infty} t_{n_{j}} & =\limsup _{j \rightarrow \infty}\left(1-\frac{\cos d(u, p)}{\sin d\left(u, T x_{n_{j}}\right) \tan \left(\frac{\alpha_{n_{j}}}{2} d\left(u, T x_{n_{j}}\right)\right)+\cos d\left(u, T x_{n_{j}}\right)}\right) \\
& =\limsup _{j \rightarrow \infty}\left(1-\frac{\cos d(u, p)}{0+\cos d\left(u, T x_{n_{j}}\right)}\right) \\
& \leq 0 .
\end{aligned}
$$

By Lemma 2.2, we have that $\lim _{n \rightarrow \infty} s_{n}=0$, that is, $\left\{x_{n}\right\}$ converges to $p=P_{F(T)} u$, and we finish the proof.

\section{Application to the image recovery problem}

In the setting of Hilbert spaces, the image recovery problem can be formulated as to find the nearest point in the intersection of a family of closed convex subsets from a given point by using the metric projection of each subset. In this section, we consider this problem in the setting of complete CAT(1) spaces. As the simplest case, we deal with only two closed convex subsets $C_{1}$ and $C_{2}$ such that $C_{1} \cap C_{2} \neq \emptyset$ and generate an iterative sequence converging to the nearest point in $C_{1} \cap C_{2}$ from a given point.

First, we observe some properties of the metric projection defined on a CAT(1) space. Let $X$ be a complete CAT(1) space, $C$ a nonempty closed $\pi$-convex subset of $X$ and suppose that $d(x, C)=\inf _{y \in C} d(x, y)<\pi / 2$ for every $x \in X$. Then we can prove that the metric projection $P: X \rightarrow C$ is a strongly quasinonexpansive and $\Delta$ demiclosed mapping such that $F\left(P_{C}\right)=C$. Indeed, it is known that $P_{C}$ is quasinonexpansive; see [14]. Let $\left\{x_{n}\right\}$ be a sequence in $X$ and $p \in C$ such that $\sup _{n \in \mathbb{N}} d\left(x_{n}, p\right)<\pi / 2$ and $\lim _{n \rightarrow \infty}\left(\cos d\left(x_{n}, p\right) / \cos d\left(P_{C} x_{n}, p\right)\right)=1$. Then, from the property of metric projection, we have that

$$
\cos d\left(x_{n}, P_{C} x_{n}\right) \cos d\left(P_{C} x_{n}, p\right) \geq \cos d\left(x_{n}, p\right)
$$

for every $n \in \mathbb{N}$. Therefore, we have

$$
1 \geq \cos d\left(x_{n}, P_{C} x_{n}\right) \geq \frac{\cos d\left(x_{n}, p\right)}{\cos d\left(P_{C} x_{n}, p\right)}
$$


and thus $\lim _{n \rightarrow \infty} \cos d\left(x_{n}, P_{C} x_{n}\right)=1$, that is, $\lim _{n \rightarrow \infty} d\left(x_{n}, P_{C} x_{n}\right)=0$. Hence, $P_{C}$ is strongly quasinonexpansive.

On the other hand, let $\left\{x_{n}\right\}$ be such that $\lim _{n \rightarrow \infty} d\left(x_{n}, P_{C} x_{n}\right)=0$ and assume that $\left\{x_{n}\right\}$ is $\Delta$-convergent to $x_{\infty} \in X$. Then $\left\{P_{C} x_{n}\right\}$ is also $\Delta$-convergent to $x_{\infty}$. Since $\left\{P_{C} x_{n}\right\}$ is a sequence in a closed $\pi$-convex subset $C$, we have that its $\Delta$-limit $x_{\infty}$ belongs to $C$, that is, $x_{\infty} \in F\left(P_{C}\right)[14]$. It shows that $P_{C}$ is $\Delta$-demiclosed.

For two strongly quasinonexpansive and $\Delta$-demiclosed mappings having common fixed points, we can create a new strongly quasinonexpansive and $\Delta$-demiclosed mapping whose fixed points are common fixed points of given two mappings. For example, as we have seen above, metric projections to closed and convex sets are strongly quasinonexpansive and $\Delta$-demiclosed. Thus, for given two metric projections to closed convex sets whose intersection is nonempty, the following method is applicable. It is useful to solve the image recovery problem.

Lemma 4.1 Let $X$ be a CAT(1) space and $y_{0}, y_{1}$ and $y$ elements of $X$ such that $d\left(y_{0}, y\right)+$ $d\left(y_{1}, y\right)+d\left(y_{0}, y_{1}\right)<2 \pi$. Then

$$
\cos d\left(\frac{1}{2} y_{0} \oplus \frac{1}{2} y_{1}, y\right) \cos \frac{d\left(y_{0}, y_{1}\right)}{2} \geq \min \left\{\cos d\left(y_{0}, y\right), \cos d\left(y_{1}, y\right)\right\}
$$

Proof It is obvious if $y_{0}=y_{1}$. Otherwise, we have that

$$
\begin{aligned}
\cos d\left(\frac{1}{2} y_{0} \oplus \frac{1}{2} y_{1}, y\right) \sin d\left(y_{0}, y_{1}\right) & \geq\left(\cos d\left(y_{0}, y\right)+\cos d\left(y_{1}, y\right)\right) \sin \frac{d\left(y_{0}, y_{1}\right)}{2} \\
& \geq 2 \min \left\{\cos d\left(y_{0}, y\right), \cos d\left(y_{1}, y\right)\right\} \sin \frac{d\left(y_{0}, y_{1}\right)}{2}
\end{aligned}
$$

Dividing above by $2 \sin \left(d\left(y_{0}, y_{1}\right) / 2\right)$, we get the conclusion.

Corollary 4.2 Let $T_{0}$ and $T_{1}$ be quasinonexpansive mappings from $X$ to $X, x_{0}$ and $x_{1}$ elements of $X$, and $p$ an element of $F\left(T_{0}\right) \cap F\left(T_{1}\right)$. Then

$$
\cos d\left(\frac{1}{2} T_{0} x \oplus \frac{1}{2} T_{1} x, p\right) \cos \frac{d\left(T_{0} x, T_{1} x\right)}{2} \geq \cos d(x, p)
$$

Lemma 4.3 Let $X$ be a complete CAT(1) space such that $d\left(\nu, v^{\prime}\right)<\pi / 2$ for arbitrary $v$ and $v^{\prime}$ of $X$, and $T_{0}$ and $T_{1}$ quasinonexpansive mappings from $X$ to $X$ such that $F\left(T_{0}\right) \cap F\left(T_{1}\right) \neq \emptyset$. Then

$$
F\left(\frac{1}{2} T_{0} \oplus \frac{1}{2} T_{1}\right)=F\left(T_{0}\right) \cap F\left(T_{1}\right)
$$

Proof It is obvious that $F\left(\frac{1}{2} T_{0} \oplus \frac{1}{2} T_{1}\right) \supset F\left(T_{0}\right) \cap F\left(T_{1}\right)$. We will show the opposite inclusion. We denote $T=\frac{1}{2} T_{0} \oplus \frac{1}{2} T_{1}$. Let $z \in F(T)$. Then, for arbitrary $p \in F\left(T_{0}\right) \cap F\left(T_{1}\right)$, from Corollary 4.2, we have that

$$
\cos d(z, p) \sin \frac{d\left(T_{0} z, T_{1} z\right)}{2}=\cos d(T z, p) \sin \frac{d\left(T_{0} z, T_{1} z\right)}{2} \geq \cos d(z, p)
$$

that is, $T_{0} z=T_{1} z$. Hence, $z=T z=T_{0} z=T_{1} z$, which means $z \in F\left(T_{0}\right) \cap F\left(T_{1}\right)$. 
Lemma 4.4 Let $X$ be a CAT(1) space such that $d\left(v, v^{\prime}\right)<\pi / 2$ for arbitrary $v$ and $v^{\prime}$ of $X$. Let $T_{0}$ and $T_{1}$ be mappings from $X$ to $X$ such that $F\left(T_{0}\right) \cap F\left(T_{1}\right) \neq \emptyset$. If both $T_{0}$ and $T_{1}$ are strongly quasinonexpansive, then so is $\frac{1}{2} T_{0} \oplus \frac{1}{2} T_{1}$.

Proof We denote $T=\frac{1}{2} T_{0} \oplus \frac{1}{2} T_{1}$. By Corollary 4.2, for $x \in X$ and $p \in F\left(T_{0}\right) \cap F\left(T_{1}\right)$, we have

$$
\cos d(T x, p) \geq \cos d(T x, p) \cos \frac{d\left(T_{0} x, T_{1} x\right)}{2} \geq \cos d(x, p),
$$

that is, $d(T x, p) \leq d(x, p)$ and hence $T$ is quasinonexpansive. Moreover, for a sequence $\left\{x_{n}\right\}$ in $X$ and a point $p$ in $F(T)$ such that $\sup _{n \in \mathbb{N}} d\left(x_{n}, p\right)<\pi / 2$ and $\lim _{n \rightarrow \infty}\left(\cos d\left(x_{n}, p\right) /\right.$ $\left.\cos d\left(T x_{n}, p\right)\right)=1$, by Lemma 4.1 , we have

$$
\begin{aligned}
\cos d\left(T x_{n}, p\right) & \geq \cos d\left(T x_{n}, p\right) \cos \frac{d\left(T_{0} x_{n}, T_{1} x_{n}\right)}{2} \\
& \geq \min \left\{\cos d\left(T_{0} x_{n}, p\right), \cos d\left(T_{1} x_{n}, p\right)\right\} .
\end{aligned}
$$

So, there exist two disjoint subsets $\left\{m_{i}\right\}$ and $\left\{n_{i}\right\}$ of $\mathbb{N}$ such that $\mathbb{N}=\left\{m_{i}\right\} \cup\left\{n_{i}\right\}$ and

$$
\begin{aligned}
& d\left(T x_{m_{i}}, p\right) \leq d\left(T_{0} x_{m_{i}}, p\right) \quad \text { for all } m_{i} \quad \text { and } \\
& d\left(T x_{n_{i}}, p\right) \leq d\left(T_{1} x_{n_{i}}, p\right) \quad \text { for all } n_{i} .
\end{aligned}
$$

We may assume that both $\left\{m_{i}\right\}$ and $\left\{n_{i}\right\}$ are infinite sets without loss of generality. From Lemma 4.3, $p$ is in $F\left(T_{0}\right)$ and thus

$$
1 \geq \frac{\cos d\left(x_{m_{i}}, p\right)}{\cos d\left(T_{0} x_{m_{i}}, p\right)} \geq \frac{\cos d\left(x_{m_{i}}, p\right)}{\cos d\left(T x_{m_{i}}, p\right)} \rightarrow 1,
$$

which means $\lim _{i \rightarrow \infty}\left(\cos d\left(x_{m_{i}}, p\right) / \cos d\left(T_{0} x_{m_{i}}, p\right)\right)=1$. Since $T_{0}$ is strongly quasinonexpansive, we have that $\lim _{i \rightarrow \infty} d\left(T_{0} x_{m_{i}}, x_{m_{i}}\right)=0$. By Corollary 4.2, we have

$$
\cos \frac{d\left(T_{0} x_{m_{i}}, T_{1} x_{m_{i}}\right)}{2} \geq \frac{\cos d\left(x_{m_{i}}, p\right)}{\cos d\left(T x_{m_{i}}, p\right)} \rightarrow 1
$$

that is, $\lim _{i \rightarrow \infty} d\left(T_{0} x_{m_{i}}, T_{1} x_{m_{i}}\right)=0$. Then it follows that $\lim _{i \rightarrow \infty} d\left(T_{1} x_{m_{i}}, x_{m_{i}}\right)=0$. Similarly, we have that $\lim _{i \rightarrow \infty} d\left(T_{1} x_{n_{i}}, x_{n_{i}}\right)=0$ and $\lim _{i \rightarrow \infty} d\left(T_{0} x_{n_{i}}, x_{n_{i}}\right)=0$. Consequently, we have that $\lim _{n \rightarrow \infty} d\left(T_{0} x_{n}, x_{n}\right)=0$ and $\lim _{n \rightarrow \infty} d\left(T_{1} x_{n}, x_{n}\right)=0$. Hence, we obtain that $\lim _{n \rightarrow \infty} d\left(T x_{n}, x_{n}\right)=0$, which is the desired result.

Lemma 4.5 Let $X$ be a CAT(1) space such that $d\left(v, v^{\prime}\right)<\pi / 2$ for arbitrary $v$ and $v^{\prime}$ of $X$. Let $T_{0}$ and $T_{1}$ be mappings from $X$ to $X$ such that $F\left(T_{0}\right) \cap F\left(T_{1}\right) \neq \emptyset$. If both $T_{0}$ and $T_{1}$ are $\Delta$-demiclosed, then so is $\frac{1}{2} T_{0} \oplus \frac{1}{2} T_{1}$.

Proof We denote $T=\frac{1}{2} T_{0} \oplus \frac{1}{2} T_{1}$. Let $\left\{x_{n}\right\}$ be a sequence in $X$ and $x_{\infty}$ an element of $X$ such that $d\left(T x_{n}, x_{n}\right) \rightarrow 0$ and suppose that $\left\{x_{n}\right\}$ is $\Delta$-convergent to $x_{\infty}$. Then, by Corollary 4.2, we have

$$
\cos \frac{d\left(T_{0} x_{n}, T_{1} x_{n}\right)}{2} \geq \frac{\cos d\left(x_{n}, p\right)}{\cos d\left(T x_{n}, p\right)} \rightarrow 1,
$$


that is, $\lim _{n \rightarrow \infty} d\left(T_{0} x_{n}, T_{1} x_{n}\right)=0$. Thus we have

$$
d\left(T_{0} x_{n}, T x_{n}\right) \leq \frac{d\left(T_{0} x_{n}, T_{1} x_{n}\right)}{2} \rightarrow 0
$$

Since $T_{0}$ is $\Delta$-demiclosed, we have that $T_{0} x_{\infty}=x_{\infty}$. In a similar fashion, we have that $T_{1} x_{\infty}=x_{\infty}$. Hence $T x_{\infty}=x_{\infty}$, that is, $T$ is $\Delta$-demiclosed.

Let $X$ be a complete CAT(1) space such that $d\left(\nu, v^{\prime}\right)<\pi / 2$ for every $v, v^{\prime} \in X$, and let $C_{0}$ and $C_{1}$ be closed convex subsets of $X$ having the nonempty intersection. Then, for the metric projections $P_{C_{0}}$ and $P_{C_{1}}$, the mapping $\frac{1}{2} P_{C_{0}} \oplus \frac{1}{2} P_{C_{1}}$ is strongly quasinonexpansive and $\Delta$-demiclosed. Moreover, the set of its fixed points is $C_{0} \cap C_{1}$. Applying these facts to Theorem 3.2, we obtain the following result for the image recovery problem for two convex subsets.

Theorem 4.6 Let $X$ be a complete CAT(1) space such that $d\left(v, v^{\prime}\right)<\pi / 2$ for every $v, v^{\prime} \in X$. Let $C_{0}$ and $C_{1}$ be closed convex subsets of $X$ such that $C_{0} \cap C_{1} \neq \emptyset$. Let $\left\{\alpha_{n}\right\}$ be a real sequence in ] 0,1 [ such that $\lim _{n \rightarrow \infty} \alpha_{n}=0$ and $\sum_{n=0}^{\infty} \alpha_{n}=\infty$. For given points $u, x_{0} \in X$, let $\left\{x_{n}\right\}$ be the sequence in $X$ generated by

$$
x_{n+1}=\alpha_{n} u \oplus\left(1-\alpha_{n}\right)\left(\frac{1}{2} P_{C_{0}} x_{n} \oplus \frac{1}{2} P_{C_{1}} x_{n}\right)
$$

for $n \in \mathbb{N}$. Suppose that one of the following conditions holds:

(a) $\sup _{v, v^{\prime} \in X} d\left(v, v^{\prime}\right)<\pi / 2$;

(b) $d\left(u, P_{C_{0} \cap C_{1}} u\right)<\pi / 4$ and $d\left(u, P_{C_{0} \cap C_{1}} u\right)+d\left(x_{0}, P_{C_{0} \cap C_{1}} u\right)<\pi / 2$;

(c) $\sum_{n=0}^{\infty} \alpha_{n}^{2}=\infty$.

Then $\left\{x_{n}\right\}$ converges to $P_{C_{0} \cap C_{1}} u$.

\section{Approximation to a fixed point of nonexpansive mappings}

At the end of this paper, we prove two convergence theorems of iterative schemes which approximate a fixed point of a nonexpansive mapping. Firstly, we apply the main result Theorem 3.2 to this problem. We begin with the following lemmas.

Lemma 5.1 A nonexpansive mapping defined on a CAT(1) space is $\Delta$-demiclosed.

Proof Let $S: X \rightarrow X$ be a nonexpansive mapping. Let $\left\{x_{n}\right\}$ be a $\Delta$-convergent sequence in $X$ with the $\Delta$-limit $x_{\infty} \in X$ and suppose that $\lim _{n \rightarrow \infty} d\left(x_{n}, S x_{n}\right)=0$. We will prove that $x_{\infty}=S x_{\infty}$. If $x_{\infty} \neq S x_{\infty}$, then, by the uniqueness of the asymptotic center, we have that

$$
\begin{aligned}
\limsup _{n \rightarrow \infty} d\left(x_{n}, x_{\infty}\right) & <\limsup _{n \rightarrow \infty} d\left(x_{n}, S x_{\infty}\right) \\
& \leq \limsup _{n \rightarrow \infty}\left(d\left(x_{n}, S x_{n}\right)+d\left(S x_{n}, S x_{\infty}\right)\right) \\
& \leq \limsup _{n \rightarrow \infty}\left(d\left(x_{n}, S x_{n}\right)+d\left(x_{n}, x_{\infty}\right)\right) \\
& =\limsup _{n \rightarrow \infty} d\left(x_{n}, x_{\infty}\right)
\end{aligned}
$$

a contradiction. Hence, we have that $S$ is $\Delta$-demiclosed. 
Lemma 5.2 Let $X$ be a CAT(1) space such that $d\left(v^{\prime}, v^{\prime \prime}\right)+d\left(v^{\prime \prime}, v\right)+d\left(v, v^{\prime}\right)<2 \pi$ for every $v, v^{\prime}, v^{\prime \prime} \in X$. Let $S: X \rightarrow X$ be a nonexpansive mapping with a nonempty set of fixed points $F(S)$. Then the mapping $T: X \rightarrow X$ defined by

$$
T x=\frac{1}{2} x \oplus \frac{1}{2} S x
$$

for $x \in X$ is a strongly quasinonexpansive and $\Delta$-demiclosed mapping such that $F(T)=$ $F(S)$.

Proof It is obvious that $F(T)=F(S)$ by definition and, since both the identity mapping $I$ and $S$ are quasinonexpansive, for $x \in X$ and $p \in F(T)=F(S)$, we have that

$$
\cos d(T x, p) \geq \frac{1}{2} \cos d(x, p)+\frac{1}{2} \cos d(S x, p) \geq \cos d(x, p) .
$$

Thus $T$ is quasinonexpansive. Let $\left\{x_{n}\right\}$ be a sequence in $X$ such that $\sup _{n \in \mathbb{N}} d\left(x_{n}, p\right)<\pi / 2$, and suppose that $\lim _{n \rightarrow \infty}\left(\cos d\left(x_{n}, p\right) / \cos d\left(T x_{n}, p\right)\right)=1$. Then we have

$$
\begin{aligned}
\cos d\left(T x_{n}, p\right) \sin d\left(x_{n}, S x_{n}\right) & \geq \sin \frac{d\left(x_{n}, S x_{n}\right)}{2}\left(\cos d\left(x_{n}, p\right)+\cos d\left(S x_{n}, p\right)\right) \\
& \geq 2 \sin \frac{d\left(x_{n}, S x_{n}\right)}{2} \cos d\left(x_{n}, p\right)
\end{aligned}
$$

for every $n \in \mathbb{N}$. It follows that

$$
\cos d\left(T x_{n}, p\right) \cos \frac{d\left(x_{n}, S x_{n}\right)}{2} \geq \cos d\left(x_{n}, p\right)
$$

and since $\sup _{n \in \mathbb{N}} d\left(T x_{n}, p\right) \leq \sup _{n \in \mathbb{N}} d\left(x_{n}, p\right)<\pi / 2$, we have

$$
\begin{aligned}
1 & \geq \limsup _{n \rightarrow \infty} \cos d\left(x_{n}, T x_{n}\right) \\
& \geq \liminf _{n \rightarrow \infty} \cos d\left(x_{n}, T x_{n}\right) \\
& =\liminf _{n \rightarrow \infty} \cos \frac{d\left(x_{n}, S x_{n}\right)}{2} \\
& \geq \lim _{n \rightarrow \infty} \frac{\cos d\left(x_{n}, p\right)}{\cos d\left(T x_{n}, p\right)} \\
= & 1 .
\end{aligned}
$$

It implies that $\lim _{n \rightarrow \infty} d\left(x_{n}, T x_{n}\right)=0$ and hence $T$ is strongly quasinonexpansive.

For the $\Delta$-demiclosedness of $T$, use Lemmas 4.5 and 5.1 with the fact that the identity mapping is also $\Delta$-demiclosed.

Applying this lemma and the results in the previous section to Theorem 3.2, we obtain the following convergence theorem of an iterative scheme approximating a fixed point of a nonexpansive mapping.

Theorem 5.3 Let $X$ be a complete CAT(1) space such that $d\left(v, v^{\prime}\right)<\pi / 2$ for every $v, v^{\prime} \in X$. Let $S: X \rightarrow X$ be a nonexpansive mapping and suppose that $F(S) \neq \emptyset$. Let $\left\{\alpha_{n}\right\}$ be a real 
sequence in $] 0,1\left[\right.$ such that $\lim _{n \rightarrow \infty} \alpha_{n}=0$ and $\sum_{n=0}^{\infty} \alpha_{n}=\infty$. For given points $u, x_{0} \in X$, let $\left\{x_{n}\right\}$ be the sequence in $X$ generated by

$$
x_{n+1}=\alpha_{n} u \oplus\left(1-\alpha_{n}\right)\left(\frac{1}{2} x_{n} \oplus \frac{1}{2} S x_{n}\right)
$$

for $n \in \mathbb{N}$. Suppose that one of the following conditions holds:

(a) $\sup _{v, v^{\prime} \in X} d\left(v, v^{\prime}\right)<\pi / 2$;

(b) $d\left(u, P_{F(S)} u\right)<\pi / 4$ and $d\left(u, P_{F(S)} u\right)+d\left(x_{0}, P_{F(S)} u\right)<\pi / 2$;

(c) $\sum_{n=0}^{\infty} \alpha_{n}^{2}=\infty$.

Then $\left\{x_{n}\right\}$ converges to $P_{F(S)} u$.

The next convergence theorem of an iterative scheme on CAT(1) spaces was first proposed by Piątek [6]. The theorem deals with the Halpern-type iterative sequence. Although the result itself is correct, a part of the proof does not seem to be exact. Precisely, in the proof of the convergence theorem for the explicit iteration process, the author makes use of Xu's lemma, Lemma 2.1 in this paper. However, the conditions required for this lemma are not verified. We attempt to prove the following theorem as a supplement of the aforementioned result, and moreover, we find another coefficient condition which guarantees convergence of the iterative scheme.

Before showing the result, we need the following lemma which is analogous to [6, Lemma 3.3]. The assumption for the length of the edges of the triangle is improved.

Lemma 5.4 Let $X$ be a CAT(1) space. For $M \in] 0, \pi[$, let $u, v, w \in X$ be such that $d(u, v) \leq M$ and $d(u, w) \leq M$. For a given $\alpha \in] 0,1\left[\right.$, let $v^{\prime}=\alpha u \oplus(1-\alpha) v$ and $w^{\prime}=\alpha u \oplus(1-\alpha) w$. If $d(v, w)+d(w, u)+d(u, v)<2 \pi$ and $\sin ((1-\alpha) M) \leq \sin M$, then

$$
d\left(v^{\prime}, w^{\prime}\right) \leq \frac{\sin ((1-\alpha) M)}{\sin M} d(v, w) .
$$

Proof Consider the comparison triangle $\Delta(\bar{u}, \bar{v}, \bar{w})$ of $\triangle(u, v, w)$ on $\mathbb{S}^{2}$ and let $\bar{v}^{\prime}$ and $\bar{w}^{\prime}$ be the comparison points of $v^{\prime}$ and $w^{\prime}$, respectively. Let

$$
U=d_{\mathbb{S}^{2}}(\bar{v}, \bar{w}), \quad V=d_{\mathbb{S}^{2}}(\bar{w}, \bar{u}), \quad W=d_{\mathbb{S}^{2}}(\bar{u}, \bar{v})
$$

and $U^{\prime}=d_{\mathbb{S}^{2}}\left(\bar{v}^{\prime}, \bar{w}^{\prime}\right)$. Then, letting $\alpha^{\prime}=1-\alpha$, we have that

$$
\begin{aligned}
\sin V & \sin W\left(\frac{\sin ^{2} \alpha^{\prime} M}{\sin ^{2} M}(1-\cos U)-\left(1-\cos U^{\prime}\right)\right) \\
= & \sin V \sin W\left(\frac{\sin ^{2} \alpha^{\prime} M}{\sin ^{2} M}(1-\cos U)-1\right) \\
& +(\cos U-\cos V \cos W) \sin \alpha^{\prime} V \sin \alpha^{\prime} W+\sin V \sin W \cos \alpha^{\prime} V \cos \alpha^{\prime} W \\
= & (1-\cos U)\left(\sin V \sin W \frac{\sin ^{2} \alpha^{\prime} M}{\sin ^{2} M}-\sin \alpha^{\prime} V \sin \alpha^{\prime} W\right) \\
& +\left((1-\cos V \cos W) \sin \alpha^{\prime} V \sin \alpha^{\prime} W-\sin V \sin W\left(1-\cos \alpha^{\prime} V \cos \alpha^{\prime} W\right)\right) .
\end{aligned}
$$


Since the functions $f_{V}(t)=\sin t V / \sin t M, f_{W}(t)=\sin t W / \sin t M$, and $g(t)=(1-\cos t V \times$ $\cos t W) /(\sin t V \sin t W)$ are all increasing on [0,1], it follows that

$$
\sin V \sin W \frac{\sin ^{2} \alpha^{\prime} M}{\sin ^{2} M}-\sin \alpha^{\prime} V \sin \alpha^{\prime} W \geq 0,
$$

and

$$
(1-\cos V \cos W) \sin \alpha^{\prime} V \sin \alpha^{\prime} W-\sin V \sin W\left(1-\cos \alpha^{\prime} V \cos \alpha^{\prime} W\right) \geq 0 .
$$

Therefore, we have that

$$
\sin V \sin W\left(\frac{\sin ^{2} \alpha^{\prime} M}{\sin ^{2} M}(1-\cos U)-\left(1-\cos U^{\prime}\right)\right) \geq 0
$$

Using the assumption that $\sin \alpha^{\prime} M \leq \sin M$, we obtain that

$$
\begin{aligned}
\sin \frac{U^{\prime}}{2} & =\sqrt{\frac{1-\cos U^{\prime}}{2}} \leq \frac{\sin \alpha^{\prime} M}{\sin M} \sqrt{\frac{1-\cos U}{2}} \\
& =\frac{\sin \alpha^{\prime} M}{\sin M} \sin \frac{U}{2} \leq \sin \left(\frac{\sin \alpha^{\prime} M}{\sin M} \frac{U}{2}\right)
\end{aligned}
$$

and, by the CAT(1) inequality, it follows that

$$
d\left(v^{\prime}, w^{\prime}\right) \leq d_{\mathbb{S}^{2}}\left(\bar{v}^{\prime}, \bar{w}^{\prime}\right)=U^{\prime} \leq \frac{\sin ((1-\alpha) M)}{\sin M} U=\frac{\sin ((1-\alpha) M)}{\sin M} d(v, w),
$$

which is the desired result.

Theorem 5.5 Let $X$ be a complete CAT(1) space such that $d\left(v, v^{\prime}\right)<\pi / 2$ for every $v, v^{\prime} \in X$. Let $T: X \rightarrow X$ be a nonexpansive mapping and suppose that $F(T) \neq \emptyset$. Let $\left\{\alpha_{n}\right\}$ be a real sequence in $[0,1]$ such that $\lim _{n \rightarrow \infty} \alpha_{n}=0, \sum_{n=0}^{\infty} \alpha_{n}=\infty$, and $\sum_{n=1}^{\infty}\left|\alpha_{n+1}-\alpha_{n}\right|<\infty$. For given points $u, x_{0} \in X$, let $\left\{x_{n}\right\}$ be the sequence in $X$ generated by

$$
x_{n+1}=\alpha_{n} u \oplus\left(1-\alpha_{n}\right) T x_{n}
$$

for $n \in \mathbb{N}$. Suppose that one of the following conditions holds:

(a) $\sup _{v, v^{\prime} \in X} d\left(v, v^{\prime}\right)<\pi / 2$;

(b) $d\left(u, P_{F(T)} u\right)<\pi / 4$ and $d\left(u, P_{F(T)} u\right)+d\left(x_{0}, P_{F(T)} u\right)<\pi / 2$;

(c) $\sum_{n=0}^{\infty} \alpha_{n}^{2}=\infty$.

Then $\left\{x_{n}\right\}$ converges to $P_{F(T)} u$.

We employ the method used in [6] for some parts of the proof.

Proof From the definition of $\left\{x_{n}\right\}$, using Lemma 5.4, we have that

$$
\begin{aligned}
d\left(x_{n}, x_{n+1}\right) & \leq d\left(x_{n}, \alpha_{n} u \oplus\left(1-\alpha_{n}\right) T x_{n-1}\right)+d\left(\alpha_{n} u \oplus\left(1-\alpha_{n}\right) T x_{n-1}, x_{n+1}\right) \\
& =\left|\alpha_{n}-\alpha_{n-1}\right| d\left(u, T x_{n-1}\right)+d\left(\alpha_{n} u \oplus\left(1-\alpha_{n}\right) T x_{n-1}, x_{n+1}\right)
\end{aligned}
$$




$$
\begin{aligned}
& \leq\left|\alpha_{n}-\alpha_{n-1}\right| d\left(u, T x_{n-1}\right)+\frac{\sin \left(\left(1-\alpha_{n}\right) M_{n}\right)}{\sin M_{n}} d\left(T x_{n-1}, T x_{n}\right) \\
& \leq\left|\alpha_{n}-\alpha_{n-1}\right| d\left(u, T x_{n-1}\right)+\frac{\sin \left(\left(1-\alpha_{n}\right) M_{n}\right)}{\sin M_{n}} d\left(x_{n-1}, x_{n}\right),
\end{aligned}
$$

where $M_{n}=\max \left\{d\left(u, T x_{n}\right), d\left(u, T x_{n-1}\right)\right\}$ for each $n \in \mathbb{N}$. Let

$$
\gamma_{n}= \begin{cases}1-\frac{\sin \left(\left(1-\alpha_{n}\right) M_{n}\right)}{\sin M_{n}} & \left(M_{n} \neq 0\right), \\ \alpha_{n} & \left(M_{n}=0\right)\end{cases}
$$

for all $n \in \mathbb{N}$. Then, as in the proof of Theorem 3.2, we have that each of the conditions (a), (b) and (c) implies that $\sum_{n=0}^{\infty} \gamma_{n}=\infty$. In particular, in cases of (a) and (b), for $M=$ $\sup _{v, v^{\prime} \in X} d\left(v, v^{\prime}\right)$ and $M=\max \left\{2 d(u, p), d(u, p)+d\left(x_{0}, p\right)\right\}$ with $p=P_{F(T)} u$, respectively, it holds that

$$
\gamma_{n} \geq \alpha_{n} \cos M_{n} \geq \alpha_{n} \cos M
$$

and in case of (c), it holds that

$$
\gamma_{n} \geq \frac{\alpha_{n}^{2} \pi^{2}}{8}
$$

Then, by using Lemma 2.1 with the condition $\sum_{n=1}^{\infty}\left|\alpha_{n+1}-\alpha_{n}\right|<\infty$, we have that $\lim _{n \rightarrow \infty} d\left(x_{n}, x_{n+1}\right)=0$. It follows that

$$
0 \leq d\left(x_{n}, T x_{n}\right) \leq d\left(x_{n}, x_{n+1}\right)+d\left(x_{n+1}, T x_{n}\right)=d\left(x_{n}, x_{n+1}\right)+\alpha_{n} d\left(u, T x_{n}\right)
$$

and thus $\lim _{n \rightarrow \infty} d\left(x_{n}, T x_{n}\right)=0$. Let $p,\left\{s_{n}\right\},\left\{t_{n}\right\},\left\{\beta_{n}\right\}$ be as in the proof of Theorem 3.2 again. Then by Lemma 3.1, we have that

$$
s_{n+1} \leq\left(1-\beta_{n}\right) s_{n}+\beta_{n} t_{n}
$$

for every $n \in \mathbb{N}$. Since every nonexpansive mapping is $\Delta$-demiclosed, we can use the same technique as the proof of Theorem 3.2 and then we obtain that

$$
\begin{aligned}
\limsup _{n \rightarrow \infty} t_{n} & =\limsup _{n \rightarrow \infty}\left(1-\frac{\cos d(u, p)}{\sin d\left(u, T x_{n}\right) \tan \left(\frac{\alpha_{n}}{2} d\left(u, T x_{n}\right)\right)+\cos d\left(u, T x_{n}\right)}\right) \\
& \leq 0 .
\end{aligned}
$$

Consequently, we have that $\lim _{n \rightarrow \infty} s_{n}=0$ by Lemma 2.1. Hence, $\left\{x_{n}\right\}$ converges to $p=$ $P_{F(T)} u$, which is the desired result. 
Author details
'Department of Information Science, Toho University, Miyama, Funabashi, Chiba 274-8510, Japan. ${ }^{2}$ Laboratory of
Mathematics, Faculty of Engineering, Tamagawa University, Tamagawa-Gakuen, Machida, Tokyo 194-8610, Japan.

\section{Acknowledgements}

The first author is supported by Grant-in-Aid for Scientific Research No. 22540175 from the Japan Society for the Promotion of Science.

\section{Received: 25 July 2012 Accepted: 18 December 2012 Published: 9 January 2013}

\section{References}

1. Halpern, B: Fixed points of nonexpanding maps. Bull. Am. Math. Soc. 73, 957-961 (1967)

2. Wittmann, R: Approximation of fixed points of nonexpansive mappings. Arch. Math. 58, 486-491 (1992)

3. Bruck, RE, Reich, S: Nonexpansive projections and resolvents of accretive operators in Banach spaces. Houst. J. Math. 3, 459-470 (1977)

4. Bruck, RE: Random products of contractions in metric and Banach spaces. J. Math. Anal. Appl. 88, $319-332$ (1982)

5. Saejung, S: Halpern's iteration in CAT(0) spaces. Fixed Point Theory Appl. 2010, Art. ID 471781 (2010)

6. Piątek, B: Halpern iteration in CAT $(\kappa)$ spaces. Acta Math. Sin. Engl. Ser. 27, 635-646 (2011)

7. Saejung, S: Halpern's iteration in Banach spaces. Nonlinear Anal. 73, 3431-3439 (2010)

8. Aoyama, K, Kimura, Y, Kohsaka, F: Strong convergence theorems for strongly relatively nonexpansive sequences and applications. J. Nonlinear Anal. Optim. 3, 67-77 (2012)

9. Bridson, MR, Haefliger, A: Metric Spaces of Non-Positive Curvature. Grundlehren der Mathematischen Wissenschaften [Fundamental Principles of Mathematical Sciences], vol. 319. Springer, Berlin (1999)

10. Kimura, Y, Satô, K: Convergence of subsets of a complete geodesic space with curvature bounded above. Nonlinear Anal. 75, 5079-5085 (2012)

11. $\mathrm{Xu}, \mathrm{H}-\mathrm{K}$ : Another control condition in an iterative method for nonexpansive mappings. Bull. Aust. Math. Soc. 65 109-113 (2002)

12. Saejung, S, Yotkaew, P: Approximation of zeros of inverse strongly monotone operators in Banach spaces. Nonlinear Anal. 75, 742-750 (2012)

13. He, JS, Fang, DH, López, G, Li, C: Mann's algorithm for nonexpansive mappings in CAT( $\kappa)$ spaces. Nonlinear Anal. 75, 445-452 (2012)

14. Espínola, R, Fernández-León, A: CAT(k)-spaces, weak convergence and fixed points. J. Math. Anal. Appl. 353, 410-427 (2009)

doi:10.1186/1687-1812-2013-7

Cite this article as: Kimura and Satô: Halpern iteration for strongly quasinonexpansive mappings on a geodesic space with curvature bounded above by one. Fixed Point Theory and Applications 2013 2013:7.

\section{Submit your manuscript to a SpringerOpen ${ }^{\odot}$ journal and benefit from:}

- Convenient online submission

Rigorous peer review

- Immediate publication on acceptance

Open access: articles freely available online

- High visibility within the field

- Retaining the copyright to your article

Submit your next manuscript at $>$ springeropen.com 